PROCEEDINGS OF THE

AMERICAN MATHEMATICAL SOCIETY

Volume 131, Number 4, Pages 1181-1189

S 0002-9939(02)06657-1

Article electronically published on July 26, 2002

\title{
STRICTLY SINGULAR NON-COMPACT OPERATORS ON HEREDITARILY INDECOMPOSABLE BANACH SPACES
}

\author{
I. GASPARIS
}

(Communicated by N. Tomczak-Jaegermann)

\begin{abstract}
An example is given of a strictly singular non-compact operator on a Hereditarily Indecomposable, reflexive, asymptotic $\ell_{1}$ Banach space. The construction of this operator relies on the existence of transfinite $c_{0}$-spreading models in the dual of the space.
\end{abstract}

\section{INTRODUCTION}

A Banach space is said to be Hereditarily Indecomposable (H.I.) if for every pair $Y, Z$ of subspaces of $X$ with $Y \cap Z=\{\mathbf{0}\}$, the subspace $Y+Z$ is not closed (by a subspace of a Banach space we shall mean an infinite dimensional, closed linear subspace). The first example of an H.I. space was given by Gowers and Maurey [16] providing a negative solution to the famous unconditional basic sequence problem. The following important result was established in [16]: Every operator on a complex H.I. space is a strictly singular perturbation of a multiple of the identity (by the term operator we shall mean a bounded linear operator). Actually, the following characterization of complex H.I. spaces is given in [11: $X$ is H.I. if, and only if, every operator from a subspace of $X$ into $X$ is a strictly singular perturbation of the inclusion map. We recall that an operator on a Banach space is strictly singular if no restriction of it to a subspace is an isomorphism.

There has been an interest in investigating strictly singular operators on H.I. spaces because of their connection to the invariant subspace problem. Indeed, known results [10], 19] yield that if $X$ is an H.I. space with the property that every strictly singular operator on $X$ is compact, then every operator on $X$ admits a non-trivial invariant subspace. It is therefore natural to investigate whether or not the known examples of H.I. spaces admit strictly singular, non-compact operators. Gowers [15] constructed an example of a strictly singular non-compact operator from a certain subspace of the Gowers-Maurey space into the whole space. An example of an operator (unpublished) with analogous properties was constructed by Argyros and Wagner on the Argyros-Deliyanni H.I. space 4 .

Recently, Androulakis and Schlumprecht 3 gave an example of a strictly singular non-compact operator on the Gowers-Maurey space. We also note that examples of H.I. spaces admitting strictly singular non-compact operators were obtained by Argyros and Felouzis as a consequence of their deep dichotomy result [7].

Received by the editors July 2, 2001 and, in revised form, November 14, 2001.

2000 Mathematics Subject Classification. Primary 46B03; Secondary 06A07, 03E02.

Key words and phrases. Hereditarily Indecomposable space, Tsirelson's space, Schreier sets.

(C)2002 American Mathematical Society 
In the present paper we show that certain asymptotic $\ell_{1}$ H.I. spaces constructed in [14] also admit strictly singular non-compact operators. This will be a consequence of the fact, established here, that their duals admit $c_{0}^{\omega}$-spreading models. We recall the definition which requires the concept of the Schreier families $\left\{S_{\xi}\right\}_{\xi<\omega_{1}}$ [1] (defined in the next section).

Definition 1.1. Suppose that $X$ is a Banach space with a basis $\left(e_{i}\right)$. A seminormalized block basis $\left(x_{i}\right)$ of $\left(e_{i}\right)$ is a $c_{0}^{\omega}$ (resp. $\ell_{1}^{\omega}$ )-spreading model if there exists a constant $C>0$ such that the following property is satisfied: For every $j \in \mathbb{N}$, every finite subset $F$ of $\mathbb{N}$ with $\min F \geq j$ and such that $\left(x_{i}\right)_{i \in F}$ is $S_{j}$-admissible, we have that $\left\|\sum_{i \in F} a_{i} x_{i}\right\| \leq C \max _{i \in F}\left|a_{i}\right|\left(\right.$ resp. $\left.\left\|\sum_{i \in F} a_{i} x_{i}\right\| \geq C \sum_{i \in F}\left|a_{i}\right|\right)$ for every scalar sequence $\left(a_{i}\right)_{i \in F}$.

The Banach spaces discussed in this paper are Tsirelson-type spaces defined as the completion of $c_{00}$ (the space of finitely supported real sequences) under norms given by suitable subsets of $\mathcal{P}$ (the set of finitely supported signed measures $\mu$ on $\mathbb{N}$ such that $|\mu(\{n\})| \leq 1$ for all $n \in \mathbb{N})$.

A subset $\mathcal{N}$ of $\mathcal{P}$ is said to be norming provided it satisfies the following:

(1) $e_{n}^{*} \in \mathcal{N}$, for all $n \in \mathbb{N}$, where $e_{n}^{*}$ denotes the point mass measure at $n$.

(2) $\mathcal{N}$ is symmetric, that is, if $\mu \in \mathcal{N}$, then $-\mu \in \mathcal{N}$.

(3) $\mathcal{N}$ is closed under restriction to intervals, that is, if $\mu \in \mathcal{N}$, then $\mu \mid J \in \mathcal{N}$, for every interval $J$ in $\mathbb{N}$.

The term norming is justified by the fact that one can define a norm $\|\cdot\|_{\mathcal{N}}$ on $c_{00}$ in the following manner:

$$
\left\|\sum_{i=1}^{\infty} a_{i} e_{i}\right\|_{\mathcal{N}}=\sup \left\{\sum_{i=1}^{\infty} a_{i} \mu(\{i\}): \mu \in \mathcal{N}\right\}
$$

for every finitely supported scalar sequence $\left(a_{i}\right)$. Of course, $\left(e_{i}\right)$ is the natural basis of $c_{00}$. Letting $X_{\mathcal{N}}$ denote the completion of $\left(c_{00},\|\cdot\|_{\mathcal{N}}\right)$, we see that $\left(e_{n}\right)$ is a normalized, bimonotone basis for $X_{\mathcal{N}}$.

We shall next describe sufficient conditions on $\mathcal{N}$ in order for $X_{\mathcal{N}}^{*}$ to admit $c_{0}^{\omega}$-spreading models. We shall be using two infinite subsets $M=\left(m_{i}\right)_{i=0}^{\infty}$ and $N=\left(n_{i}\right)_{i=0}^{\infty}$ of $\mathbb{N}$ satisfying the following requirements:

$$
\begin{aligned}
& m_{0}>1 \text { and there exists an increasing sequence of integers }\left(s_{i}\right)_{i=0}^{\infty} \\
& \text { so that } m_{j}=\prod_{i<j} m_{i}^{s_{i}} \text { for all } j \geq 1
\end{aligned}
$$

$4 f_{j}<n_{j}$ for all $j \geq 0$, where $\left(f_{j}\right)$ is defined as follows: $f_{0}=1$, while for $j \geq 1, f_{j}=\max \left\{\sum_{i<j} \rho_{i} n_{i}: \rho_{i} \in \mathbb{N} \cup\{0\}(i<j), \prod_{i<j} m_{i}^{\rho_{i}}<m_{j}\right\}$.

In order to state our result we need to introduce some notation.

Notation. $\quad(1)$ Given $\mu, \nu$ in $\mathcal{P}$, we write $\mu<\nu$ if $\max \operatorname{supp} \mu<\min \operatorname{supp} \nu$.

(2) A finite subset $A$ of $\mathcal{P}$ is $S_{p^{-}}$admissible, $p \in \mathbb{N}$, if $A=\left\{\mu_{1}<\cdots<\mu_{k}\right\}$ and $\left\{\min \operatorname{supp} \mu_{i}: i \leq k\right\} \in S_{p}$.

(3) Given $\mathcal{N} \subset \mathcal{P}$ and $j \in \mathbb{N} \cup\{0\}$ we set $\mathcal{N}_{j}=\left\{\left(1 / m_{j}\right) \sum_{\mu \in A} \mu: A \subset\right.$ $\mathcal{N}$ is $S_{n_{j}}-$ admissible\}. 
(4) $\mathcal{N}_{\infty}=\left\{\theta \sum_{i=1}^{k} \mu_{i}: k \in \mathbb{N}, \theta \in\left(0,1 / m_{0}\right], \mu_{1}<\cdots<\mu_{k}, \exists \tau:\{1, \ldots, k\} \rightarrow\right.$ $\left.\mathbb{N} \cup\{0\}, 1-1, \mu_{i} \in \mathcal{N}_{\tau(i)}(i \leq k)\right\}$.

The following definition will be important for our purposes.

Definition 1.2. A norming set $\mathcal{N}$ is said to be $(M, N)$-Schreier if the following properties are satisfied:

(1) $\mathcal{N} \subset \bigcup_{i=0}^{\infty} \mathcal{N}_{i} \cup \mathcal{N}_{\infty} \cup\left\{ \pm e_{n}^{*}: n \in \mathbb{N}\right\}$.

(2) $\mathcal{N}_{i} \subset \mathcal{N}$, for $0 \leq i<\infty$.

The natural norming set of the mixed Tsirelson space $T\left(\frac{1}{m_{i}}, S_{n_{i}}\right)_{i=0}^{\infty}$, turns out to be $(M, N)$-Schreier. Further, one can check that if $N=\left(n_{i}\right)_{i=0}^{\infty}$ is $M$-good (this term is defined in [14] $)\left(M=\left(m_{i}\right)_{i=0}^{\infty}\right)$ and $M^{(2)}=\left(m_{2 i}\right)_{i=0}^{\infty}, N^{(2)}=\left(n_{2 i}\right)_{i=0}^{\infty}$ satisfy conditions (1.1), (1.2), then the norming set $\mathcal{N}$ of the H.I. space $X_{\mathcal{N}}$ constructed in [14] is $\left(M^{(2)}, N^{(2)}\right)$-Schreier.

The main result of this paper is the following:

Theorem 1.3. Suppose $\mathcal{N}$ is $(M, N)$-Schreier. Then $X_{\mathcal{N}}^{*}$ admits a $c_{0}^{\omega}$-spreading model $\left(x_{i}^{*}\right)$. Moreover, for a suitably chosen infinite sequence of integers $\left(l_{i}\right)$, there exists a non-compact operator $T$ on $X_{\mathcal{N}}$ satisfying $T x=\sum_{i=1}^{\infty} x_{l_{i}}^{*}(x) e_{i}$ for all $x \in X_{\mathcal{N}}$. In case $X_{\mathcal{N}}$ is H.I., then $T$ can, in addition, be taken to be strictly singular.

In fact, under the assumptions of Theorem [1.3 given any sequence $\left(a_{i}\right)$ in $\ell_{\infty}$ the formula $S x=\sum_{i=1}^{\infty} a_{i} x_{l_{i}}^{*}(x) e_{i}, x \in X_{\mathcal{N}}$, defines an operator on $X_{\mathcal{N}}$. It follows from this that the space of operators on $X_{\mathcal{N}}$ contains a subspace isomorphic to $\ell_{\infty}$ (cf. [3]).

An immediate consequence of Theorem 1.3 is our next corollary.

Corollary 1.4. Let $M, N$ be infinite subsets of $\mathbb{N}$ subject to conditions (1.1) and (1.2). Then the dual of the mixed Tsirelson space $T\left(\frac{1}{m_{i}}, S_{n_{i}}\right)_{i=0}^{\infty}$ admits a $c_{0}^{\omega}$ spreading model.

Applying Theorem 1.3 to the H.I. space $X_{\mathcal{N}}$ constructed in [14] we obtain:

Corollary 1.5. There exists an asymptotic $\ell_{1}$, reflexive H.I. Banach space that admits a strictly singular non-compact operator and whose dual admits a $c_{0}^{\omega}$-spreading model.

Remark. In an earlier version of this paper we actually showed that the dual of every subspace of the H.I. space constructed in [14] admits a $c_{0}^{\omega}$-spreading model.

Standard duality arguments yield that if $X_{\mathcal{N}}^{*}$ admits a $c_{0}^{\omega}$-spreading model, then $X_{\mathcal{N}}$ admits an $\ell_{1}^{\omega}$-spreading model. However, the converse is not true in general. Thus, our approach provides a new method of showing that certain mixed Tsirelson spaces admit $\ell_{1}^{\omega}$-spreading models. This problem has been studied in [6] where it is shown that every subspace of certain regular mixed Tsirelson spaces 2 contains an $\ell_{1}^{\omega}$-spreading model. Their approach is based on the finite representability of $c_{0}$ in such spaces [5]. The method of constructing $c_{0}^{\omega}$-spreading models in $X_{\mathcal{N}}^{*}$ relies on the existence of normalized functionals in $\mathcal{N}$ which belong simultaneously to different classes $\mathcal{N}_{j}, j \geq 0$. Our method is also applied in [6] in order to show that certain modified mixed Tsirelson spaces [5] also admit $\ell_{1}^{\omega}$-spreading models. We finally mention the result of D. Kutzarova and P.K. Lin [17] on the existence of $\ell_{1}$-spreading models in Schlumprecht's space [22]. 


\section{Preliminaries}

We shall make use of standard Banach space facts and terminology as may be found in [18]. Let $X$ be a Banach space. A sequence $\left(x_{n}\right)$ in $X$ is semi-normalized if there exists $\delta>0$ such that $\delta \leq\left\|x_{n}\right\| \leq 1$, for all $n$.

Given any set $D$, we let $[D]$ (resp. $D^{<\infty}$ ) denote the set of its infinite (resp. finite) subsets. Given $M \in[\mathbb{N}]$, the notation $M=\left(m_{i}\right)$ indicates that $M=\left\{m_{1}<\right.$ $\left.m_{2}<\cdots\right\}$. Let $E$ and $F$ be finite subsets of $\mathbb{N}$. We write $E<F$ if $\max E<\min F$.

Suppose now that $X$ has a Schauder basis $\left(e_{n}\right)$. A sequence $\left(u_{n}\right)$ of non-zero vectors in $X$ is a block basis of $\left(e_{n}\right)$ if there exist successive subsets $F_{1}<F_{2}<\cdots$ of $\mathbb{N}$ and a scalar sequence $\left(a_{n}\right)$ so that $u_{n}=\sum_{i \in F_{n}} a_{i} e_{i}$, for every $n \in \mathbb{N}$. We adopt the notation $u_{1}<u_{2}<\cdots$ to indicate that $\left(u_{n}\right)$ is a block basis of $\left(e_{n}\right)$. We let supp $u_{n}$ denote the set $\left\{i \in F_{n}: a_{i} \neq 0\right\}$.

We shall next review the Schreier hierarchy $\left\{S_{\xi}\right\}_{\xi<\omega_{1}}$ [1]. Since we shall only be using the families $\left\{S_{\xi}\right\}_{\xi<\omega}$, we confine the definitions to the finite ordinal case.

The Schreier families. We let $S_{0}=\{\{n\}: n \in \mathbb{N}\} \cup\{\emptyset\}$. Suppose $S_{\xi}$ has been defined, $\xi<\omega$. We set

$$
S_{\xi+1}=\left\{\bigcup_{i=1}^{n} F_{i}: n \in \mathbb{N}, n \leq \min F_{1}, F_{1}<\cdots<F_{n}, F_{i} \in S_{\xi}(i \leq n)\right\} \cup\{\emptyset\} .
$$

An important property shared by the Schreier families is that they are hereditary: If $F \in S_{\xi}$ and $G \subset F$, then $G \in S_{\xi}$. Another important property is that they are spreading: If $\left\{p_{1}, \cdots, p_{k}\right\} \in S_{\xi}, p_{1}<\cdots<p_{k}$, and $q_{1}<\cdots<q_{k}$ are so that $p_{i} \leq q_{i}$ for all $i \leq k$, then $\left\{q_{1}, \cdots, q_{k}\right\} \in S_{\xi}$. It is not hard to check that if $F_{1}<\cdots<F_{n}$ are members of $S_{\alpha}$ such that $\left\{\min F_{i}: i \leq n\right\}$ belongs to $S_{\beta}$, then $\bigcup_{i=1}^{n} F_{i}$ belongs to $S_{\alpha+\beta}$.

A finite collection $\mathcal{F}$ of finite subsets of $\mathbb{N}$ is said to be $S_{\xi}$-admissible, $\xi<\omega$, if there exists an enumeration $\left\{I_{k}: k \leq n\right\}$ of $\mathcal{F}$ such that $I_{1}<\cdots<I_{n}$ and the set $\left\{\min I_{k}: k \leq n\right\}$ is a member of $S_{\xi}$. A finite block basis $u_{1}<\cdots<u_{n}$ in a Banach space with a basis is $S_{\xi}$-admissible if $\left\{\operatorname{supp} u_{i}: i \leq n\right\}$ is also. A Banach space $X$ with a basis $\left(e_{n}\right)$ is asymptotic $\ell_{1}[20$ if there exists $\delta>0$ such that every $S_{1}$-admissible block basis $\left(u_{i}\right)_{i=1}^{p}$ of $\left(e_{n}\right)$ satisfies $\left\|\sum_{i=1}^{p} u_{i}\right\| \geq \delta \sum_{i=1}^{p}\left\|u_{i}\right\|$.

\section{Tree Representations of FUnCtionals in $\mathcal{N}$}

In this section we describe tree representations of members of $\mathcal{N}$ which turn out to be very useful in estimating the norm of certain functionals in $\mathcal{N}$.

We recall that a tree is a partially ordered finite set $(\mathcal{T}, \leq)$, such that for every $\alpha \in \mathcal{T}$, the set $\{\beta \in \mathcal{T}: \beta \leq \alpha\}$ is well ordered. The elements of $\mathcal{T}$ are called nodes. A node of $\mathcal{T}$ is terminal if it has no successors in $\mathcal{T}$. Given $\alpha \in \mathcal{T}$ which is not terminal, we denote by $D_{\alpha}(\mathcal{T})$ the set of the immediate successors of $\alpha$ in $\mathcal{T}$. A tree $\mathcal{T}$ is rooted if it has a unique node $\alpha_{0}$ (the root) such that $\alpha_{0} \leq \alpha$ for all $\alpha \in \mathcal{T}$. A branch of $\mathcal{T}$ is a maximal, under inclusion, well ordered subset. The height $o(\mathcal{T})$ of $\mathcal{T}$ is the cardinality of its longest branch.

In the sequel, $\mathcal{N}$ is a $(M, N)$-Schreier set of measures (see Definition 1.2).

Definition 3.1. A functional tree in $\mathcal{N}$ is a subset $\left(x_{\alpha}^{*}\right)_{\alpha \in \mathcal{T}}$ of $\mathcal{N}$ indexed by a rooted tree $\mathcal{T}$ and such that the following are satisfied:

(1) $\operatorname{supp} x_{\beta}^{*} \subset \operatorname{supp} x_{\alpha}^{*}$ whenever $\alpha<\beta$ in $\mathcal{T}$. 
(2) If $\alpha \in \mathcal{T}$ is non-terminal, then $\left(x_{\beta}^{*}\right)_{\beta \in D_{\alpha}(\mathcal{T})}$ is, under an appropriate enumeration, a finite block basis of $\left(e_{n}^{*}\right)$.

Definition 3.2. Let $x^{*} \in \mathcal{N}$. A tree representation of $x^{*}$ is a functional tree $\left(x_{\alpha}^{*}\right)_{\alpha \in \mathcal{T}}$ in $\mathcal{N}$ together with a function $\psi: \mathcal{T} \rightarrow\left[m_{0}, \infty\right) \cup\{0\}$ so that the following properties hold:

(1) $x_{\alpha_{0}}^{*}=x^{*}$, where $\alpha_{0}$ is the root of $\mathcal{T}$.

(2) $\psi(\alpha)=0$ if, and only if, $\alpha$ is terminal. In that case $x_{\alpha}^{*}= \pm e_{p_{\alpha}}^{*}$ for some $p_{\alpha} \in \mathbb{N}$.

(3) If $\alpha \in \mathcal{T}$ is non-terminal, then $x_{\alpha}^{*}=(1 / \psi(\alpha)) \sum_{\beta \in D_{\alpha}(\mathcal{T})} x_{\beta}^{*}$.

(4) Every non-terminal $\alpha \in \mathcal{T}$ is either of type I or of type II. Specifically, $\alpha$ is of type I if $\psi(\alpha)=m_{j}$ and $\left(x_{\beta}^{*}\right)_{\beta \in D_{\alpha}(\mathcal{T})}$ is $S_{n_{j}}$-admissible for some $j \geq 0 . \alpha$ is of type II if $D_{\alpha}(\mathcal{T})$ consists of type I nodes and $\psi \mid D_{\alpha}(\mathcal{T})$ is $1-1$.

Notation. We set $\psi_{\alpha}=\prod_{\beta<\alpha} \psi(\beta)$ if $\alpha$ is not the root of $\mathcal{T}$, and $\psi_{\alpha}=1$ if $\alpha$ is the root of $\mathcal{T}$.

It is not hard to see that every member of $\mathcal{N}$ admits a (not necessarily unique) tree representation.

Remark. Suppose that $\left(x_{\alpha}^{*}\right)_{\alpha \in \mathcal{T}}$ is a tree representation for $x^{*}$ with associated function $\psi$. The following facts can be easily established by induction on $o(\mathcal{T})$ :

(1) Let $A$ be a subset of $\mathcal{T}$ consisting of pairwise incomparable nodes. Then $\left(x_{\alpha}^{*}\right)_{\alpha \in A}$ is, under an appropriate enumeration, a block basis of $\left(e_{n}^{*}\right)$.

(2) If $A$ is additionally assumed to intersect every branch of $\mathcal{T}$, then $x^{*}=$ $\sum_{\alpha \in A} \frac{1}{\psi_{\alpha}} x_{\alpha}^{*}$.

The proof of Theorem 1.3 requires the following lemma.

Lemma 3.3. Let $\left(x_{\alpha}^{*}\right)_{\alpha \in \mathcal{T}}$ be a functional tree in $\mathcal{N}$ and let $\phi: \mathcal{T} \rightarrow \mathbb{N}$ be a function such that if $\alpha \in \mathcal{T}$ is non-terminal, then $\left(x_{\beta}^{*}\right)_{\beta \in D_{\alpha}(\mathcal{T})}$ is $S_{\phi(\alpha)}$-admissible. Then, for every subset $A$ of $\mathcal{T}$ consisting of pairwise incomparable nodes, the collection $\left(x_{\alpha}^{*}\right)_{\alpha \in A}$ is $S_{p}$-admissible, where $p=\max \left\{\sum_{\beta<\alpha} \phi(\beta): \alpha \in A\right\}$.

Proof. The proof of the lemma is done by induction on $o(\mathcal{T})$. If $o(\mathcal{T})=1$ the assertion of the lemma is trivial. Assuming the assertion true when $o(\mathcal{T})<k, k>1$, let $\mathcal{T}$ be such that $o(\mathcal{T})=k$. Let $\alpha_{0}$ be the root of $\mathcal{T}$ and set $\mathcal{T}_{\alpha}=\{\beta \in \mathcal{T}: \alpha \leq \beta\}$, for all $\alpha \in D_{\alpha_{0}}(\mathcal{T})$. Our assumptions yield that $\left(x_{\alpha}^{*}\right)_{\alpha \in D_{\alpha_{0}}(\mathcal{T})}$ is $S_{\phi\left(\alpha_{0}\right) \text {-admissible. }}$ We can assume that $|A| \geq 2$ and set $A_{\alpha}=\mathcal{T}_{\alpha} \cap A$, for all $\alpha \in D_{\alpha_{0}}(\mathcal{T})$. Since $o\left(\mathcal{T}_{\alpha}\right)<k$ our induction hypothesis implies that $\left(x_{\beta}^{*}\right)_{\beta \in A_{\alpha}}$ is $S_{p_{\alpha}}$-admissible, where $p_{\alpha}=\max \left\{\sum_{\alpha \leq \beta<\gamma} \phi(\beta): \gamma \in A_{\alpha}\right\}$, for all $\alpha \in A$. Note that $p_{\alpha} \leq p-\phi\left(\alpha_{0}\right)$, for all $\alpha \in A$. This completes the inductive step as $\left(x_{\alpha}^{*}\right)_{\alpha \in D_{\alpha_{0}}(\mathcal{T})}$ is $S_{\phi\left(\alpha_{0}\right) \text {-admissible. }}$

\section{Proof of Theorem 1.3}

The existence of a $c_{0}^{\omega}$-spreading model $\left(x_{k}^{*}\right)$ in $X_{\mathcal{N}}^{*}$ follows after establishing Lemma 4.1 and Corollary 4.4. The former shows that a natural candidate for $\left(x_{k}^{*}\right)$ satisfies an upper $c_{0}^{\omega}$-estimate. The latter implies that this particular sequence $\left(x_{k}^{*}\right)$ is semi-normalized, based on a decomposition result (Lemma 4.3) for the elements of $\mathcal{N}$, and thus it is indeed a $c_{0}^{\omega}$-spreading model. It will be crucial for the entire proof that $x_{k}^{*} \in \mathcal{N}_{j}$, for all $j \leq k$. 
Remark. Observe that if $x_{1}^{*}<\cdots<x_{p}^{*}$ is an $S_{n_{i}+n_{j}}$-admissible sequence in $\mathcal{N}$, for some $i \geq 0, j \geq 0$, then $\frac{1}{m_{i} m_{j}} \sum_{k=1}^{p} x_{k}^{*} \in \mathcal{N}$. Indeed, we may decompose $\{1, \ldots, p\}$ into successive subsets $F_{1}<\cdots<F_{t}$ so that $\left(x_{k}^{*}\right)_{k \in F_{r}}$ is $S_{n_{j}}$-admissible for every $r \leq t$, while $\left(x_{\min F_{r}}^{*}\right)_{r=1}^{t}$ is $S_{n_{i}}$-admissible. If we set $y_{r}^{*}=\frac{1}{m_{j}} \sum_{k \in F_{r}} x_{k}^{*}$, then $y_{r}^{*} \in \mathcal{N}$ for all $r \leq t$, by condition (2) of Definition 1.2. Clearly, $\left(y_{r}^{*}\right)_{r=1}^{t}$ is $S_{n_{i}}$-admissible and thus $\frac{1}{m_{i}} \sum_{r=1}^{t} y_{r}^{*} \in \mathcal{N}$, again by condition (2) of Definition 1.2

A similar inductive argument now implies the following: Let $q=\sum_{i=0}^{t} a_{i} n_{i}$ for some $t \in \mathbb{N} \cup\{0\}$ and $a_{i} \in \mathbb{N} \cup\{0\}, i \leq t$. Assume that $\left(x_{l}^{*}\right)_{l=1}^{k}$ is an $S_{q^{-a d m i s s i b l e}}$ collection of functionals in $\mathcal{N}$. Then $\left(1 / \prod_{i \leq t} m_{i}^{a_{i}}\right) \sum_{l=1}^{k} x_{l}^{*} \in \mathcal{N}$.

Notation. We set $p_{k}=\sum_{i<k} s_{i} n_{i}$, for all $k \in \mathbb{N}$ (see (1.1)). Note that $p_{k} \leq 2 f_{k}$ by the choice of $N$.

Lemma 4.1. Let $F_{1}<F_{2}<\cdots<$ be successive subsets of $\mathbb{N}$ such that $F_{k} \in S_{p_{k}}$ and set $x_{k}^{*}=\frac{1}{m_{k}} \sum_{i \in F_{k}} e_{i}^{*}$, for all $k \in \mathbb{N}$. Assume $\left(x_{k}^{*}\right)_{k=1}^{\infty}$ is semi-normalized. Then $\left(x_{k}^{*}\right)_{k=1}^{\infty}$ is a $c_{0}^{\omega}$-spreading model.

Proof. We first observe that for all $l \leq k$ we can find $z_{1}^{*}<\cdots<z_{t}^{*}$ in $\mathcal{N}$, $S_{p_{l}}$-admissible so that $x_{k}^{*}=\frac{1}{m_{l}} \sum_{i=1}^{t} z_{i}^{*}$. Indeed, we may write $F_{k}=\bigcup_{i=1}^{t} H_{i}$, where $H_{1}<\cdots<H_{t}$ belong to $S_{p_{k}-p_{l}}$ and $\left(H_{i}\right)_{i=1}^{t}$ is $S_{p_{l}}$-admissible. Set $z_{i}^{*}=$ $\left(m_{l} / m_{k}\right) \sum_{r \in H_{i}} e_{r}^{*}, i \leq t$. But since $p_{k}-p_{l}=\sum_{l \leq j<k} s_{j} n_{j}$, and $z_{i}^{*}=\frac{1}{\prod_{l \leq j<k} m_{j}^{s_{j}}}$ $\sum_{r \in H_{i}} e_{r}^{*}, i \leq t$, our preceding remark yields that $\left(z_{i}^{*}\right)_{i=1}^{t}$ is an $S_{p_{l}}$-admissible family in $\mathcal{N}$ which clearly satisfies $x_{k}^{*}=\left(1 / m_{l}\right) \sum_{i=1}^{t} z_{i}^{*}$.

Now let $k_{0} \in \mathbb{N}$. Let $F \in \mathbb{N}^{<\infty}$ with $\min F \geq k_{0}$ and so that $\left(x_{k}^{*}\right)_{k \in F}$ is $S_{f_{k_{0}}}$-admissible. According to our initial observation, for each $k \in F$ there exists an $S_{p_{k_{0}}}$-admissible family $\left(y_{i}^{*}\right)_{i \in G_{k}}$ in $\mathcal{N}$, so that $x_{k}^{*}=\frac{1}{m_{k_{0}}} \sum_{i \in G_{k}} y_{i}^{*}$. Note that $p_{k_{0}} \leq 2 f_{k_{0}}$. We now obtain, since $4 f_{k_{0}}<n_{k_{0}}$, that $\left(y_{i}^{*}\right)_{i \in G}$ is $S_{n_{k_{0}}}$-admissible, where $G=\bigcup_{k \in F} G_{k}$. Of course, $\sum_{k \in F} x_{k}^{*}=\frac{1}{m_{k_{0}}} \sum_{i \in G} y_{i}^{*}$. Therefore, $\sum_{k \in F} x_{k}^{*} \in \mathcal{N}$, by condition (2) of Definition 1.2. The proof is now complete since $n \leq f_{n}$ for all $n \in \mathbb{N}$.

We shall also make use of the following numerical result.

Lemma 4.2. Assume that $\left(a_{i}\right)_{i=0}^{k-1}$ are positive integers satisfying $\prod_{i<k} m_{i}^{a_{i}}<m_{k}$. Then $\sum_{i<k} a_{i} n_{i}<\sum_{i<k} s_{i} n_{i}$.

Proof. By induction on $k$. The case $k=1$ is trivial since $a_{0}<s_{0}$. Assume the assertion holds for some $k \geq 1$ and let the integers $\left(a_{i}\right)_{i=0}^{k}$ satisfy $\prod_{i<k+1} m_{i}^{a_{i}}<$ $m_{k+1}$. Observe that $m_{k+1}=m_{k}^{s_{k}+1}$. Clearly, $a_{k} \leq s_{k}$. We shall distinguish between two cases: $a_{k}=s_{k}$ and $a_{k}<s_{k}$. If the former, then $\prod_{i<k} m_{i}^{a_{i}}<m_{k}$. By the induction hypothesis we obtain $\sum_{i<k} a_{i} n_{i}<\sum_{i<k} s_{i} n_{i}$. The assertion now follows as $a_{k}=s_{k}$.

When $a_{k}<s_{k}$, we obtain that $\prod_{i<k} m_{i}^{a_{i}}<m_{k}^{s_{k}-a_{k}+1}$. Our assumptions on $N$ allow us to deduce that $\sum_{i<k} a_{i} n_{i} \leq 2\left(s_{k}-a_{k}+1\right) f_{k}$. It follows that $\sum_{i<k+1} a_{i} n_{i} \leq$ $2\left(s_{k}-a_{k}+1\right) f_{k}+a_{k} n_{k}$. But also, $2\left(s_{k}-a_{k}+1\right) f_{k}+a_{k} n_{k}<s_{k} n_{k}$. Indeed, the latter inequality follows easily as $4 f_{k}<n_{k}$. Hence, $\sum_{i<k+1} a_{i} n_{i}<s_{k} n_{k}$ from which the assertion follows. The inductive step as well as the proof of the lemma are now complete. 
Lemma 4.3. Let $k \in \mathbb{N}$ and $x^{*} \in \mathcal{N}$ such that $k \leq \min \operatorname{supp} x^{*}$. Then there exist $p \in \mathbb{N}$, a partition $\left\{I_{1}, I_{2}\right\}$ of $\{1, \ldots, p\}$, functionals $x_{1}^{*}<\cdots<x_{p}^{*}$ in $\mathcal{N}$ and scalars $\left(\lambda_{i}\right)_{i=1}^{p}$ in $(0,1]$ so that the following are satisfied:

(1) $x^{*}=\sum_{i=1}^{p} \lambda_{i} x_{i}^{*}$.

(2) $x_{i}^{*}= \pm e_{j_{i}}^{*}$ for all $i \in I_{1}$ and $\left\{j_{i}: i \in I_{1}\right\} \in S_{p_{k}-1}$.

(3) $\lambda_{i} \leq 1 / m_{k}$ for all $i \in I_{2}$.

Proof. Let $\left(x_{\alpha}^{*}\right)_{\alpha \in \mathcal{T}}$ be a tree representation for $x^{*}$ with associated function $\psi$. Let $\mathfrak{B}$ denote the set of all branches of $\mathcal{T}$. Given $b \in \mathfrak{B}$ let $\alpha(b)$ denote the smallest node $\beta \in b$ such that $\psi_{\beta} \geq m_{k}$, or, if such a $\beta$ does not exist, let $\alpha(b)$ be the terminal node of $b$.

We let $A=\{\alpha(b): b \in \mathfrak{B}\}$. It is not hard to check that $A$ consists of pairwise incomparable nodes of $\mathcal{T}$. Since $A$ intersects all branches of $\mathcal{T}$, we have that $x^{*}=\sum_{\alpha \in A} \frac{1}{\psi_{\alpha}} x_{\alpha}^{*}$. We now set $A_{1}=\left\{\alpha \in A: \psi_{\alpha}<m_{k}\right\}$ and $A_{2}=A \backslash A_{1}$. It is clear that $A_{1}$ consists of terminal nodes of $\mathcal{T}$. Let $\left(x_{i}^{*}\right)_{i=1}^{p}$ be an enumeration of $\left(x_{\alpha}^{*}\right)_{\alpha \in A}$ such that $x_{1}^{*}<\cdots<x_{p}^{*}$. We now define $I_{r}=\left\{i \leq p: x_{i}^{*} \in\left(x_{\alpha}^{*}\right)_{\alpha \in A_{r}}\right\}$ for $r \leq 2$. We finally set $\lambda_{i}=1 / \psi_{\alpha}(i \leq p)$ if $x_{i}^{*}=x_{\alpha}^{*}$ for some $\alpha \in A$.

We need only show that $\left(x_{i}^{*}\right)_{i \in I_{1}}$ is $S_{p_{k}-1}$-admissible. The rest of the required properties are straightforward. To this end, we set $\mathcal{R}=\bigcup_{\alpha \in A_{1}}\{\beta \in \mathcal{T}: \beta \leq \alpha\}$. The key point is that if $\beta \in \mathcal{R}$ is of type II in $\mathcal{T}$, then $\left|D_{\beta}(\mathcal{R})\right| \leq k$. Indeed, $\psi \mid D_{\beta}(\mathcal{R})$ is $1-1$ as $\beta$ is of type II. On the other hand $\psi(\gamma)<m_{k}$ and $\psi(\gamma) \in M$ for all $\gamma \in D_{\beta}(\mathcal{R})$. It follows that $\left|D_{\beta}(\mathcal{R})\right| \leq k$. In particular, $\left(x_{\gamma}^{*}\right)_{\gamma \in D_{\beta}(\mathcal{R})}$ is $S_{1}$-admissible.

Define $\phi: \mathcal{R} \rightarrow \mathbb{N}$ by

$$
\phi(\beta)= \begin{cases}n_{i}, & \text { if } \beta \text { is of type I and } \psi(\beta)=m_{i}, \text { for some } i \geq 0, \\ n_{0}, & \text { if } \beta \text { is of type II, } \\ 1, & \text { if } \beta \in A_{1} .\end{cases}
$$

We now have that $\left(x_{\gamma}^{*}\right)_{\gamma \in D_{\beta}(\mathcal{R})}$ is $S_{\phi(\beta)}$-admissible, for every non-terminal $\beta \in \mathcal{R}$. Since $\prod_{\beta<\alpha} \psi(\beta)<m_{k}$, for all $\alpha \in A_{1}$, Lemma 4.2 yields $\sum_{\beta<\alpha} \phi(\beta)<p_{k}$, for all $\alpha \in A_{1}$. Therefore, $\left(x_{\alpha}^{*}\right)_{\alpha \in A_{1}}$ is $S_{p_{k}-1}$-admissible by Lemma 3.3 . This completes the entire proof.

Corollary 4.4. For every $x^{*} \in \mathcal{N}$ and $k \in \mathbb{N}$ we have that $\left\{i \in \mathbb{N}, i \geq k:\left|x^{*}\left(e_{i}\right)\right| \geq\right.$ $\left.2 / m_{k}\right\}$ belongs to $S_{p_{k}-1}$.

Proof. We can assume that min $\operatorname{supp} x^{*} \geq k$. We next apply Lemma 4.3 to conclude that $\left\{i \in \mathbb{N}, i \geq k:\left|x^{*}\left(e_{i}\right)\right| \geq 2 / m_{k}\right\}$ is contained in $\left\{j_{i}: i \leq p\right\}$ which belongs to $S_{p_{k}-1}$.

Proof of Theorem 1.3. We first show that given $k, l$ in $\mathbb{N}$ there exists $F_{k} \in S_{p_{k}}$, $l<\min F_{k}$, so that letting $x_{k}^{*}=\frac{1}{m_{k}} \sum_{i \in F_{k}} e_{i}^{*}$ we have that $1 / 3 \leq\left\|x_{k}^{*}\right\| \leq 1$. Once this is accomplished and since we can take the $F_{k}$ 's to be successive, it will follow from Lemma 4.1 that $\left(x_{k}^{*}\right)$ is a $c_{0}^{\omega}$-spreading model.

A standard property of the repeated averages hierarchy [9] (Lemma 2.3 of [13]) allows us to find $F_{k} \in S_{p_{k}}$ with $\max \{k, l\}<\min F_{k}$, positive scalars $\left(a_{i}\right)_{i \in F_{k}}$ with $\sum_{i \in F_{k}} a_{i}=1$ and such that $\sum_{i \in G} a_{i}<\frac{1}{m_{k}}$ for every $G \in S_{p_{k}-1}$. It follows readily from Corollary 4.4 that for every $x^{*} \in \mathcal{N}$, the set $\left\{i \in F_{k}:\left|x^{*}\left(e_{i}\right)\right| \geq 2 / m_{k}\right\}$ belongs to $S_{p_{k}-1}$. Letting $x_{k}=\sum_{i \in F_{k}} a_{i} e_{i}$, we conclude that $\left\|x_{k}\right\| \leq \frac{3}{m_{k}}$. But since $x_{k}^{*} \in \mathcal{N}$ and $x_{k}^{*}\left(x_{k}\right)=1 / m_{k}$, we have that $\frac{1}{3} \leq\left\|x_{k}^{*}\right\| \leq 1$, as desired. 
For the moreover assertions, we may assume, by passing to a subsequence of the $x_{k}^{*}$ 's, that if $F \in \mathbb{N}^{<\infty}, \min F>j$, and $\left(x_{i}^{*}\right)_{i \in F}$ is $S_{n_{j}}$-admissible, then $\left\|\sum_{i \in F} x_{i}^{*}\right\| \leq 1$.

We define a linear map $T: c_{00} \rightarrow c_{00}$ by the formula $T x=\sum_{i} x_{i}^{*}(x) e_{i}$. We are going to show that there exists a constant $C>0$ such that $\|T x\|_{\mathcal{N}} \leq C\|x\|_{\mathcal{N}}$, for all $x \in c_{00}$. It will then follow that $T$ extends to an operator on $X_{\mathcal{N}}$, still denoted by $T$, which satisfies $T x=\sum_{i} x_{i}^{*}(x) e_{i}$, for all $x \in X_{\mathcal{N}} \cdot T$ is the desired operator. Indeed, $T$ is non-compact because $\left(x_{i}^{*}\right)$ is semi-normalized. On the other hand if $\operatorname{Ker}(T)$ is infinite-dimensional (which is the case if $\mathbb{N} \backslash \bigcup_{i} \operatorname{supp} x_{i}^{*}$ is infinite) and $X_{\mathcal{N}}$ is H.I., then $T$ is strictly singular.

It thus remains to establish that $T$ is bounded on $c_{00}$ with respect to the $\|\cdot\|_{\mathcal{N}}$ norm. To this end, fix a normalized $x \in X_{\mathcal{N}}$ and let $x^{*} \in \mathcal{N}$. If $x^{*}= \pm e_{n}^{*}$ for some $n \in \mathbb{N}$, then we easily see that $\left|x^{*}(T x)\right| \leq 1$. If the support of $x^{*}$ contains at least two elements, set $H_{1}=\left\{i \in \operatorname{supp} x^{*}: \frac{2}{m_{1}} \leq\left|x^{*}\left(e_{i}\right)\right| \leq \frac{1}{m_{0}}\right\}$ and $H_{k}=\{i \in$ $\left.\operatorname{supp} x^{*}: \frac{2}{m_{k}} \leq\left|x^{*}\left(e_{i}\right)\right|<\frac{2}{m_{k-1}}\right\}$, for $k \geq 2$. Note that $\operatorname{supp} x^{*}=\bigcup_{k=1}^{\infty} H_{k}$. We also put $G_{k}=\left\{i \in H_{k}: i \geq k\right\}$. Corollary 4.4 yields that $G_{k} \in S_{n_{k}}$, as $p_{k}<n_{k}$ for all $k \geq 1$, and therefore $\sum_{i \in G_{k}}\left|x_{i}^{*}(x)\right| \leq 2$. We now have

$$
\begin{aligned}
\left|x^{*}(T x)\right| & =\left|\sum_{k=1}^{\infty} \sum_{i \in H_{k}} x_{i}^{*}(x) x^{*}\left(e_{i}\right)\right| \\
& \leq \sum_{i \in H_{1}}\left|x_{i}^{*}(x)\right|\left|x^{*}\left(e_{i}\right)\right|+\sum_{k=2}^{\infty}\left(\sum_{i \in G_{k}}\left|x_{i}^{*}(x)\right|+\sum_{i \in H_{k} \backslash G_{k}}\left|x_{i}^{*}(x)\right|\right) \frac{2}{m_{k-1}} \\
& \leq \frac{2}{m_{0}}+\sum_{k=2}^{\infty}\left(\frac{4}{m_{k-1}}+2 \frac{k-1}{m_{k-1}}\right) \leq \sum_{k \geq 0} \frac{4+2 k}{m_{k}}=D .
\end{aligned}
$$

To complete the proof of the theorem we need only take $C=\max \{D, 1\}$.

\section{ACKNOWLEDGMENTS}

We thank Spiros Argyros for his suggestions regarding the material discussed in this paper.

\section{REFERENCES}

[1] D. Alspach and S.A. Argyros, Complexity of weakly null sequences, Dissertationes Mathematicae 321 (1992), 1-44. MR 93j:46014

[2] G. Androulakis and E. Odell, Distorting mixed Tsirelson spaces, Israel J. Math. 109 (1999), 125-149. MR 2000f: 46012

[3] G. Androulakis and Th. Schlumprecht, Strictly singular, non-compact operators exist on the space of Gowers and Maurey, J. London Math. Soc. (2) 64 (2001), 1-20.

[4] S.A. Argyros and I. Deliyanni, Examples of asymptotic $\ell_{1}$ Banach spaces, Trans. Amer. Math. Soc. 349 (1997), 973-995. MR 97f:46021

[5] S.A. Argyros, I. Deliyanni, D.N. Kutzarova and A. Manoussakis, Modified mixed Tsirelson spaces, J. Funct. Anal. 159 (1998), 43-109. MR 2000j:46031

[6] S.A. Argyros, I. Deliyanni and A. Manoussakis, Distortion and spreading models in modified mixed Tsirelson spaces, preprint.

[7] S.A. Argyros and V. Felouzis, Interpolating Hereditarily Indecomposable Banach Spaces, J. Amer. Math. Soc. 13 (2000), 243-294. MR 2002b:46021

[8] S.A. Argyros and I. Gasparis, Unconditional structures of weakly null sequences, Trans. Amer. Math. Soc. 353 (2001), no. 5, 2019-2058. MR 2002b:46012

[9] S.A. Argyros, S. Mercourakis and A. Tsarpalias, Convex unconditionality and summability of weakly null sequences, Israel J. Math. 107 (1998), 157-193. MR 99m:46021 
[10] N. Aroszajn and K.T. Smith, Invariant subspaces of completely continuous operators, Ann. of Math. (2) 60 (1954), 345-350. MR 16:488b

[11] V. Ferenczi, Operators on subspaces of hereditarily indecomposable Banach spaces, Bull. London Math. Soc. 29 (1997), no.3, 338-344. MR 98b:47028

[12] T. Figiel and W. B. Johnson, A uniformly convex Banach space which contains no $\ell_{p}$, Compositio Math. 29 (1974), 179-190. MR 50:8011

[13] I. Gasparis and D.H. Leung, On the complemented subspaces of the Schreier spaces, Studia Math. 141 (2000), no. 3, 273-300. MR 2001j:46009

[14] I. Gasparis, A continuum of totally incomparable hereditarily indecomposable Banach spaces, submitted.

[15] W.T. Gowers, A remark about the scalar-plus-compact problem, Convex geometric analysis (Berkeley, CA, 1996), 111-115. MR 99m:46015

[16] W.T. Gowers and B. Maurey, The unconditional basic sequence problem, J. Amer. Math. Soc. 6 (1993), 851-874. MR 94k:46021.

[17] D.N. Kutzarova and P.K. Lin, Remarks about Schlumprecht space, Proc. Amer. Math. Soc. 128 (2000), no. 7, 2059-2068. MR 2000m:46031

[18] J. Lindenstrauss and L. Tzafriri, Classical Banach spaces I, Springer-Verlag, New York (1977). MR 58:17766

[19] V.I. Lomonosov, Invariant subspaces for operators commuting with compact operators, Functional Anal. Appl. 7 (1973), 213-214.

[20] B. Maurey, V.D. Milman and N. Tomczak-Jaegermann, Asymptotic infinite-dimensional theory of Banach spaces, Oper. Theory: Adv. Appl. 77 (1994), 149-175. MR 97g:46015

[21] E. Odell, On subspaces, Asymptotic Structure, and Distortion of Banach Spaces; Connections with Logic, Analysis and Logic, (C. Finet and C. Michaux, eds.) (2000), 301-376 (to appear).

[22] Th. Schlumprecht, An arbitrarily distortable Banach space, Israel J. Math. 76 (1991), 81-95. MR 93h:46023

[23] J. Schreier, Ein Gegenbeispiel zur theorie der schwachen konvergenz, Studia Math. 2 (1930), $58-62$.

[24] B. S. Tsirelson, Not every Banach space contains $\ell_{p}$ or $c_{0}$, Funct. Anal. Appl. 8 (1974), p. $138-141$.

Department of Mathematics, Oklahoma State University, Stillwater, Oklahoma 74078-1058

E-mail address: ioagaspa@math.okstate.edu

Current address: Department of Mathematics, University of Crete, Knossou Avenue, P.O. Box 2208, Herakleion, Crete 71409, Greece

E-mail address: ioagaspa@math.uch.gr 